\title{
Modeling Human-Likeness in Approaching Motions of Dual-Arm Autonomous Robots
}

\author{
Néstor García, Jan Rosell and Raúl Suárez
}

\begin{abstract}
This paper addresses the problem of obtaining human-like motions with an anthropomorphic dual-arm torso assembled on a mobile platform. The focus is set on the coordinated movements of the robotic arms and the robot base while approaching a table to subsequently perform a bimanual manipulation task. For this, human movements are captured and mapped to the robot in order to compute the human dual-arm synergies. Since the demonstrated synergies change depending on the robot position, a recursive Cartesian-space discretization is presented based on these differences. Thereby, different movements of the arms are assigned to different regions of the Cartesian space. As an application example, a motion-planning algorithm exploiting this information is proposed and used.
\end{abstract}

\section{INTRODUCTION}

Robotic systems in which a mobile platform is combined with a robotic arm are commonly known as mobile manipulators. Such combined systems are able to perform dexterous manipulation tasks in larger and more cluttered workspaces than a fixed-base manipulator due to its redundancy and translating degrees of freedom (DOFs). This versatility is augmented when two robotic arms are mounted on the mobile platform (e.g. the mobile anthropomorphic dual-arm robot shown in Fig. 1). In general, dual-arm robotic systems are used to perform coordinated manipulation tasks including regrasping [1], either arriving to a closed kinematic chain (e.g. assembling a nut and a bolt [2]), or cooperating with open chain coordinated movements (e.g. a dual-arm system using both hands to remove potential obstacles in order to obtain free access to a desired object [3]). Even though dual-arm robot manipulation has been widely investigated, it still belongs to the most demanding challenges in robotics [4]. However, to fully use the advantages offered by a mobile manipulator, it is necessary to understand how to properly and effectively plan its motions.

Nowadays, robots are getting more and more DOFs, making the motion-planning harder to solve since the dimension of problem also increases. These problems are typically solved using sampling-based planning algorithms, with the Probabilistic Road Map (PRM) and Rapidly-exploring Random Tree (RRT) being two of the most commonly used [5]. These algorithms have been researched extensively. Hence, several variants exist to deal with constraints [6], to

The authors are with the Institute of Industrial and Control Engineering (IOC), Universitat Politècnica de Catalunya (UPC), Barcelona, Spain (\{nestor.garcia.hidalgo, jan.rosell, raul.suarez $\} @$ upc.edu). This work was partially supported by the Spanish Government through the projects DPI2013-40882-P and DPI2016-80077-R. N. García is supported by the Generalitat de Catalunya through the grant FI-DGR 2017.

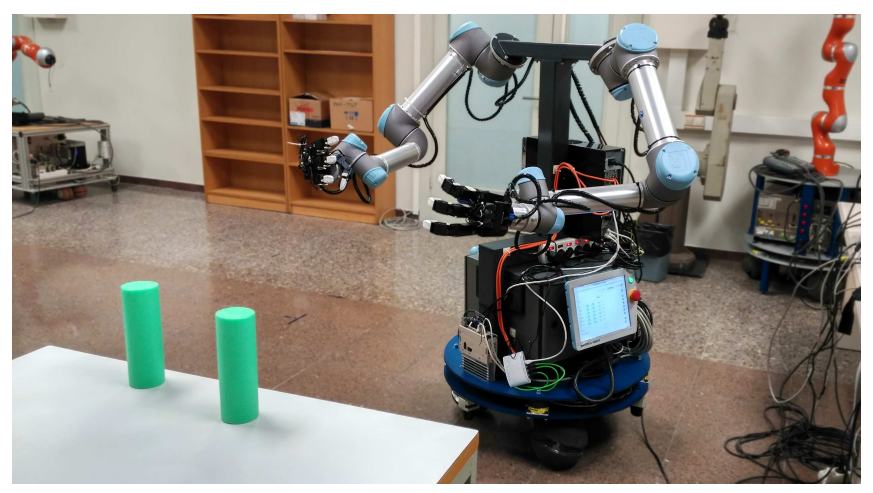

Fig. 1. Mobile anthropomorphic dual-arm robot approaching a table to perform a dual-arm manipulation task.

consider configuration-space cost-maps [7], or to bias the sampling towards better regions of the configuration space by using, potential fields [8] or retraction-based methods [9].

The approaches dealing with the coordinated motion of mobile manipulators attempted first the motion planning in two levels: first, the pose of the end effector of the manipulator was obtained and then the pose of the mobile platform was selected to optimize a specific performance function (e.g. maximizing the manipulability of the robotic arm [10]). However, in order to use the full redundancy of the robotic system, other works used a whole body planner to solve the motion planning in the complete configuration space of the robot. For instance, the RRT path planning approach was adapted and combined with inverse kinematics algorithms for motion planning along given end-effector paths [11]. The PRM algorithm was also adapted to plan task-consistent collision-free motions for mobile manipulators [12].

Other well-known approaches are based on the imitation of human motions, which is mainly formulated as non-linear optimization problems [13]. This faces another important issue: in the last decade, robots have been getting closer to humans, introducing consequently the necessity for anthropomorphic movements to allow better and safer human-robot interactions (i.e. humans can predict more easily anthropomorphic robot motions thus avoiding collisions and enhancing the collaboration with the robot [14]).

So as to obtain human-like motions, relevant works dealt with the grasping problem studying the correlations of the finger joints when the human was grasping objects [15]. These correlations were called hand postural synergies. There exist other approaches that compute the synergies from hand movements when trying to cover the whole hand 
configuration space in an unconstrained way [16]. These synergies can be used then to simplify the motion planning by reducing the dimension of the search space as well as to mimic human postures [17]. More recently, a compliant model, called soft synergies, was also introduced and used in the selection of grasping forces to control the motion of grasped objects [18]. In addition, the synergies were used in a dual-arm anthropomorphic system while performing manipulation tasks [19], [20]. All these works dealt with synergies involving correlations between joint positions but the concept of postural synergies was also extended to the space of the first derivative of the configuration trajectories [21]. These synergies, called first-order synergies, were recently also applied in dual-arm manipulation tasks [22] and used for human-likeness evaluation purposes [23].

The human arm movements have been studied in handpointing motions [24] and hand-reaching motions [25], [26]. Nevertheless, the movements of the arms have not been investigated yet in dual-arm tasks neither used in the motion planning of bimanual tasks using a mobile base. Hence, this opens a completely new field of research that is addressed in this work.

After this introduction, the paper is structured as follows. First, the problem statement and the approach overview are introduced in Section II. Then, the proposal is detailed through Sections III, IV and V. Finally, Section VI presents the experimentation and Section VII concludes the paper with the conclusions and the future work.

\section{Problem Statement and Approach Overview}

This work studies how the position and the orientation of the robot base affects the dual-arm synergies of a mobile anthropomorphic dual-arm robot. The final goal is to find a human-like coordination of the robot translational movements and the arms movements while approaching a table to perform a dual-arm manipulation task. This coordination can then be exploited in the motion-planning process, e.g. to improve its performance or to obtain human-like motions. The main features of the proposed approach are the following:

1) Human movements are captured and then mapped to the robotic system while a human operator walks towards a table and solves manipulation tasks there (see Section III). Note that this work is not focused on biped motions, but only on the upper-limb movements.

2) The captured movements are analyzed to extract the correlations between the robot position, its orientation and the configuration of the arms. The variations of these correlations are studied and the dual-arm synergies are computed from the mapped robot configurations. Then, the Cartesian space is discretized into different regions based on the changes in the computed synergies and the observed correlations (see Section IV).

3) As an application example, a motion-planning algorithm is introduced that takes profit of the synergies in the different regions of the Cartesian space, such that the coordinated movements of the arms is similar to the ones of a human being (see Section V).

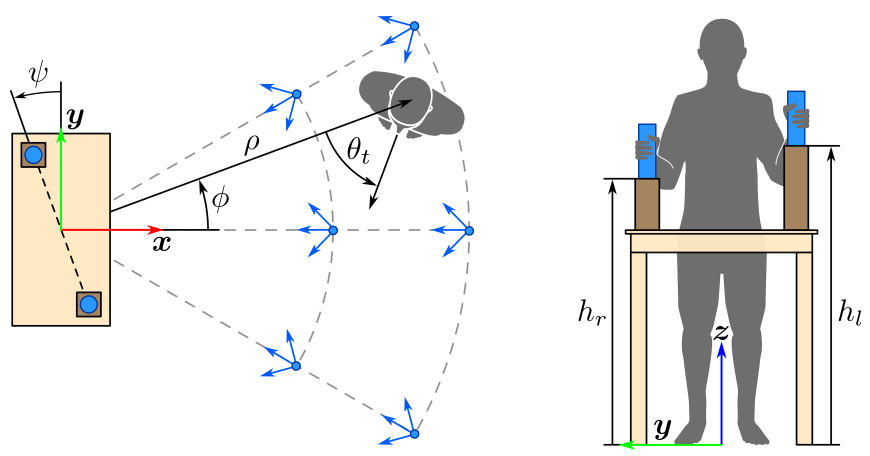

Fig. 2. Experiment performed to capture the human movements: Top view of the start time (left) and front view of the end time (right). The layout of the start positions and orientations is also shown (but not in scale).

\section{MOTION CAPTURE AND MAPPING}

\section{A. Experimental setup}

The setup used in the experimentation consists of:

- A mobile anthropomorphic dual-arm robot composed of two 6-DOF robotic arms UR5 (see Fig. 1). The arms are assembled, emulating the human-arm configuration, on a custom-designed omnidirectional mobile platform with three spherical wheels. In turn, each arm is equipped with a 16-DOF Allegro Hand.

- An optical motion-capture system formed by reflective tracking markers and 16 infrared OptiTrack cameras, allowing a 3D localization of the markers with submillimeter accuracy with a rate of $100 \mathrm{~Hz}$.

- A simulation tool, called The Kautham Project [27], with capabilities for collision checking, motion planning and graphical visualization of the whole system.

\section{B. Motion capture}

In this work, human motions are used as a reference to obtain human-like motions for mobile dual-arm manipulators. The focus is set on the approaching movements previous to the execution of bimanual manipulation tasks. For this purpose, the movements of a human operator walking towards a table and grasping two cylinders placed on pedestals have been captured. The human operator starts the experiment standing in a position and orientation parametrized by the distance $\rho$, the azimuth angle $\phi$ and the torso angle $\theta_{t}$; and the positions of the cylinders are determined by the heights $h_{l}$ and $h_{r}$ and the angle $\psi$ (see Fig. 2). Note that the final position and orientation of the human operator is not fixed, it is only required to face the table and grasp both cylinders at the same time. Since it is not computationally feasible to cover the whole parameter space, the following illustrative values have been independently used in the experiments: $\rho \in\{2,3\} \mathrm{m}, \phi \in\left\{-\frac{\pi}{6}, 0, \frac{\pi}{6}\right\} \mathrm{rad}, \theta_{t} \in\left\{-\frac{\pi}{4}, 0, \frac{\pi}{4}\right\} \mathrm{rad}$, $\psi \in\left\{-\frac{\pi}{6}, 0, \frac{\pi}{6}\right\} \mathrm{rad}$ and $h_{l}, h_{r} \in\{1,1.5\} \mathrm{m}$. This makes a total of 216 different experiments in which the 3D position of the shoulders, elbows and wrists have been recorded using markers placed on the human arms (see Fig. 3). In addition, two more markers have been attached to each palm to help in the computation of the wrist orientations. 


\section{Motion mapping}

Once the human movements have been captured, they must be mapped to the robotic system taking into account:

1) functional constraints, i.e. the robotic wrists should be positioned and oriented similarly as the human wrists.

2) anthropomorphic requirements, i.e. the complete pose of the robot should look similar to the human pose.

For manipulation tasks, the exact reproduction of the wrist positions is more important than the human-likeness of the robot configuration. Thereby, the mapping of the human movements is formulated here as a constrained optimization problem where the second observation above is subordinated to the first one. For this, let first:

- $\chi=(x, y) \in \mathbb{R}^{2}$ be a given position of the robot base, expressed in a given world reference system (see Fig. 3).

- $\boldsymbol{\theta}=\left[\theta_{t}, \boldsymbol{\theta}_{l}, \boldsymbol{\theta}_{r}\right]^{\top} \in \mathbb{R}^{1+2 n}$ be a torso configuration, i.e. the concatenation of the value of the torso angle $\theta_{t}$ (see Fig. 2) and the joint values $\boldsymbol{\theta}_{l}$ and $\boldsymbol{\theta}_{r}$ of the left and right arms (where $n$ is the number of DOFs of each robotic arm, which is 6 for the used robot).

- $\mathcal{C} \subseteq \mathbb{R}^{3+2 n}$ be the whole robot configuration space and $\boldsymbol{q}=[\boldsymbol{\chi}, \boldsymbol{\theta}]^{\top} \in \mathcal{C}$ be a configuration of the robot.

- $\mathcal{C}_{\text {free }} \subseteq \mathcal{C}$ be the subspace of the configurations $\boldsymbol{q} \in \mathcal{C}$ in which the robot is not in collision (neither with itself nor with the environment).

In addition, for each arm $i \in\{l, r\}$, with $l$ and $r$ standing for the left and right arms respectively, let:

- $\boldsymbol{p}_{\mathrm{W}_{i}}^{\mathrm{h}}, \boldsymbol{p}_{\mathrm{W}_{i}} \in \mathbb{R}^{3}$ be, respectively, the positions of the human and robotic wrists, with respect to a given world reference frame (see Fig. 3).

- $R_{\mathrm{W}_{i}}^{\mathrm{h}}, R_{\mathrm{W}_{i}} \in S O(3)$ be, respectively, the rotation matrices of the human and robotic wrists, expressed in a common reference system. Note that $R_{\mathrm{W}_{i}}^{\mathrm{h}}$ is computed from the three markers in the human palm and wrist.

- $E_{i} \geq 0$ be the tracking error of the robotic wrist, considering both position and orientation differences with respect to the human wrist (see Fig. 3 and 4). Given a weight $\lambda \in(0,1)$ balancing the relative importance of the position and orientation errors, $E_{i}$ is computed as

$$
E_{i}=\lambda\left\|\boldsymbol{p}_{\mathrm{W}_{i}}^{\mathrm{h}}-\boldsymbol{p}_{\mathrm{W}_{i}}\right\|^{2}+(1-\lambda)\left\|\ln \left(R_{\mathrm{W}_{i}}^{\mathrm{T}} R_{\mathrm{W}_{i}}^{\mathrm{h}}\right)\right\|_{F}^{2}
$$

Note that the more similar the poses of the robotic and human wrists are, the lower $E_{i}$ is (reaching its minimum value 0 if both $\boldsymbol{p}_{\mathrm{W}_{i}}=\boldsymbol{p}_{\mathrm{W}_{i}}^{\mathrm{h}}$ and $R_{\mathrm{W}_{i}}=R_{\mathrm{W}_{i}}^{\mathrm{h}}$ hold [28]).

- $A_{i} \geq 0$ be the anthropomorphic dissimilarity between the poses of the robotic arm and the human arm (i.e. the whole kinematic chains and not only the wrist poses). For this, a given number $m$ of points $\boldsymbol{p}_{j_{i}}$ are selected along the robotic arm (from the shoulder to the wrist) and also their kinematically equivalent points $\boldsymbol{p}_{j_{i}}^{\mathrm{h}}$ on the human arm (see Fig. 3 and 4). Then, $A_{i}$ is computed as the weighted sum of the squared distances between the paired $\boldsymbol{p}_{j_{i}}^{\mathrm{h}}$ and $\boldsymbol{p}_{j_{i}}$, i.e. given some positive weights $\omega_{j}$

$$
A_{i}=\sum_{j=1}^{m} \omega_{j}\left\|\boldsymbol{p}_{j_{i}}^{\mathrm{h}}-\boldsymbol{p}_{j_{i}}\right\|^{2}
$$

Hence, as $A_{i}$ decreases, the human-likeness of the robot configuration improves. Here, $m=9$ points $\boldsymbol{p}_{j_{i}}$ were

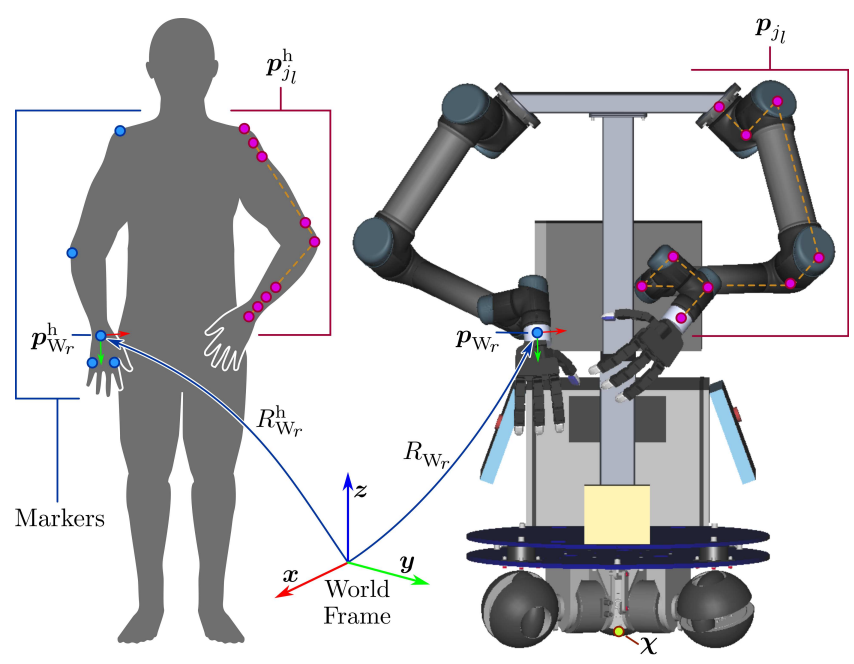

Fig. 3. Human operator and mobile anthropomorphic dual-arm robot: markers on the human arm; points $\boldsymbol{p}_{j_{i}}$ on the robotic arm with their equivalent points $\boldsymbol{p}_{j_{i}}^{\mathrm{h}}$ on the human arm; wrist positions $\boldsymbol{p}_{\mathrm{W}_{i}}$ and $\boldsymbol{p}_{\mathrm{W}_{i}}^{\mathrm{h}}$; wrist orientations $R_{\mathrm{W}_{i}}$ and $R_{\mathrm{W}_{i}}^{\mathrm{h}}$; and position $\chi$ of the robot base.

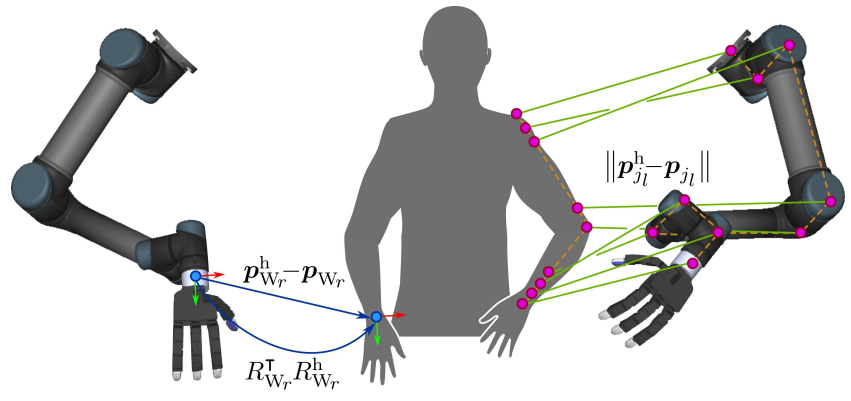

Fig. 4. Position and orientation differences in the tracking error $E_{i}$ (left), and distances involved in the anthropomorphic dissimilarity $A_{i}$ (right). Despite showing the robotic arms free-flying, $E_{i}$ and $A_{i}$ are computed for both arms with the robot assembled and sharing the same base pose $\chi$.

chosen matching them up with the intersections of the cylinders forming the robotic arm (see Fig. 3). Note that only the shoulder, elbow and wrist points are actually captured from the human arm. The other points $\boldsymbol{p}_{j_{i}}^{\mathrm{h}}$ have been selected along the shoulder-elbow and elbow-wrist rectilinear segments such that, in each segment, the ratio $\left\|\boldsymbol{p}_{j+1_{i}}-\boldsymbol{p}_{j_{i}}\right\| /\left\|\boldsymbol{p}_{j+1_{i}}^{\mathrm{h}}-\boldsymbol{p}_{j_{i}}^{\mathrm{h}}\right\|$ is constant at each sampling time.

Notice that the dependence on time and $\boldsymbol{q}$ in the above descriptions has been omitted to simplify the used notation.

Then, given the position in 3D space of the markers on the human operator, the mapping problem involves finding a configuration $\boldsymbol{q}^{*} \in \mathcal{C}_{\text {free }}$ (i.e. collision-free) that minimizes the anthropomorphic dissimilarity while guaranteeing at the same time the minimum tracking error between the human and the robot. This can be formalized as follows

$$
\begin{aligned}
\boldsymbol{q}^{*}= & \underset{\boldsymbol{q} \in \mathcal{C}_{\text {free }}}{\arg \min } A_{l}(\boldsymbol{q})+A_{r}(\boldsymbol{q}) \\
& \text { s.t. } \quad E_{l}(\boldsymbol{q})+E_{r}(\boldsymbol{q}) \leq E_{l}(\tilde{\boldsymbol{q}})+E_{r}(\tilde{\boldsymbol{q}}) \quad \forall \tilde{\boldsymbol{q}} \in \mathcal{C}_{\text {free }}
\end{aligned}
$$

Note that finding local minima is good enough for the considered purposes and that, in most cases, a perfect tracking is feasible (i.e. $E_{l}=E_{r}=0$ ). In addition to this, if 
closed-form solutions are available for the inverse kinematics of the robotic arms, then the dimension of the optimization error is reduced by 12 , with the consequent speed up. Moreover, to speed up the collision checking, simplified models of the robot and the environment (based on boxes, spheres and capsules) have been used. Thereby, the proposed mapping procedure ensures the correct placement of the robotic wrists and, simultaneously, it handles the robot redundancy by maximizing its human-likeness. Nevertheless, the human-likeness is dependent on the differences in size and kinematic structure of the robot with respect the human (i.e. smaller robots, for instance, may have to extend the arms more than the human does to reach the desired wrist poses, leading to maybe not so human-like arm configurations).

\section{Motion ANALYSIS}

The dual-arm synergies (i.e. couplings between DOFs) are obtained running a Principal Component Analysis over the set of torso configurations mapped from the human. This returns a new basis of the torso configuration space, whose axes are sorted in decreasing order of the associated sample variance (i.e. the first axis marks the direction with maximum sample variance and so on). Each axis is called a synergy and the motion along it, equivalent to a single DOF, implies the movement of several (or all) joints. This simple linear approximation is enough to capture the subspace where the demonstrated motions lie. It has been demonstrated to be useful and implementable by a drive mechanism [29] or a real-time algorithm [30]. However, nonlinear approaches to obtain synergies have been also proposed (e.g. [15]).

The dual-arm synergies depend on the robot position $\chi$. Therefore, the Cartesian space is recursively partitioned into sectors of annuli centered on the table. The splitting radii $\rho$ and angles $\phi$ are chosen such that the dual-arm synergies associated to each annular sector are significantly different to the ones from the neighboring sectors (see below). Thereby, given the parent set $Q$ of robot configurations $q$ lying within a given annular sector being partitioned, let:

- $Q_{\rho}^{-}=\left\{\boldsymbol{q} \in Q \mid x^{2}+y^{2} \leq \rho^{2}\right\}$ and $Q_{\rho}^{+}=\left\{\boldsymbol{q} \in Q \mid x^{2}+y^{2}>\rho^{2}\right\}$ be the descendant sets of $Q$ (if splitting the sector by $\rho$ ) in which the robot is respectively closer/farther to the table than a given distance $\rho$.

- $Q_{\phi}^{-}=\{\boldsymbol{q} \in Q \mid y \leq x \tan \phi\}$ and $Q_{\phi}^{+}=\{\boldsymbol{q} \in Q \mid y>x \tan \phi\}$ be the descendant sets of $Q$ (if splitting the sector by $\phi$ ) in which the robot is seen from the table more on the right/left than a given azimuth angle $\phi$, respectively.

Let a partition dividing a sector be valid if each descendant sector contains at least 5000 configurations of $Q$ and has an aspect ratio less than 1:5, i.e. the longer side of the resulting sector measures in the $\rho-\phi$ space measures at most five times the shorter side. These values have been empirically chosen. Nevertheless, the sensibility of the procedure with respect to these parameters is not high, thus their values are not a critical issue. In addition, let the likeness $\mathcal{L}$ of two sets $Q_{A}$ and $Q_{B}$, taking into account only the torso configurations, be defined as the overlapping of the distributions of the configurations in the sets [20]. $\mathcal{L}$ can be computed as

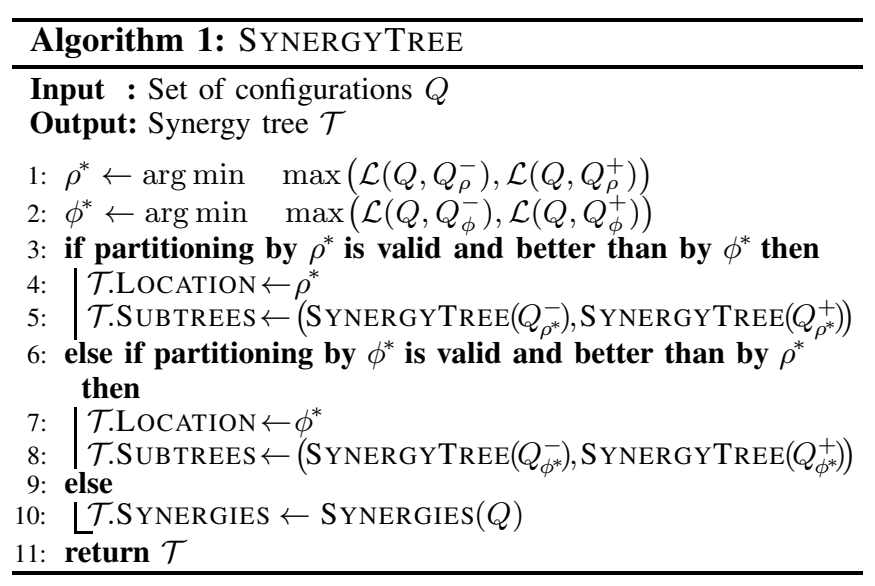

$$
\mathcal{L}\left(Q_{A}, Q_{B}\right)=\frac{e^{-\frac{1}{2}\left(\boldsymbol{\mu}_{A}-\boldsymbol{\mu}_{B}\right)^{\top}\left(\Sigma_{A}+\Sigma_{B}\right)^{-1}\left(\boldsymbol{\mu}_{A}-\boldsymbol{\mu}_{B}\right)}}{\sqrt{(2 \pi)^{1+2 n}\left|\Sigma_{A}+\Sigma_{B}\right|}}
$$

where $\boldsymbol{\mu}_{A}$ and $\boldsymbol{\mu}_{B}$ are the barycenters and $\Sigma_{A}$ and $\Sigma_{B}$ are the covariance matrices of the torso configurations in $Q_{A}$ and $Q_{B}$, respectively. Then, the best position to divide a sector is the one that minimizes the objective function $f$ defined as the maximum likeness between the parent set $Q$ and its descendant sets (i.e. $f=\max \left(\mathcal{L}\left(Q, Q_{\rho}^{-}\right), \mathcal{L}\left(Q, Q_{\rho}^{+}\right)\right)$if splitting by $\rho$, or $f=\max \left(\mathcal{L}\left(Q, Q_{\phi}^{-}\right), \mathcal{L}\left(Q, Q_{\phi}^{+}\right)\right)$if splitting by $\phi)$. Therefore, a given partition is better than others, if the others are non-valid or have a greater value of $f$.

The proposed Cartesian-space discretization is stored into a synergy tree, which is a $k$-d tree structure containing:

a) non-leaf nodes, with the partition location (i.e. whether the sector is split by $\rho$ or by $\phi$ and at which value) and the synergy subtrees before and after the partition.

b) leaf nodes, composed of the dual-arm synergies of the mapped movements lying in the corresponding sector.

The synergy tree is built by recursively applying the next procedure (outlined in Algorithm 1). First, the best partition distance $\rho^{*}$ and angle $\phi^{*}$ are computed (Lines 1-2). Then, the sector is split up by the best valid partition, among the ones defined by $\rho^{*}$ (Lines 3-5) and $\phi^{*}$ (Lines 6-8), if a valid partition exists. This procedure is recursively self-invoked until no valid partitions are found (Lines 9-10).

The introduced partition procedure has been applied to the set of configurations containing the data captured in Section III together with this same data reflected in the $y$-axis of the world frame, see Fig. 2, in order to artificially increase the number of samples. Thereby, the partition of the Cartesian-space shown in Fig. 5 is obtained, which is formed by 10 symmetrically-distributed annular sectors. Notice that the synergies are similar in regions far from the table independently of $\phi$ (i.e. one big sector exists in which the arms are mostly at resting). While getting closer to the table, the dual-arm synergies differ and are grouped into different sectors, remarking the gradual transition from the free-walk to the grasping phase (i.e. the arms get ready to reach the goal pose). Finally, when the robot is in front of the table grasping the cylinders, a unique set of synergies exists. 


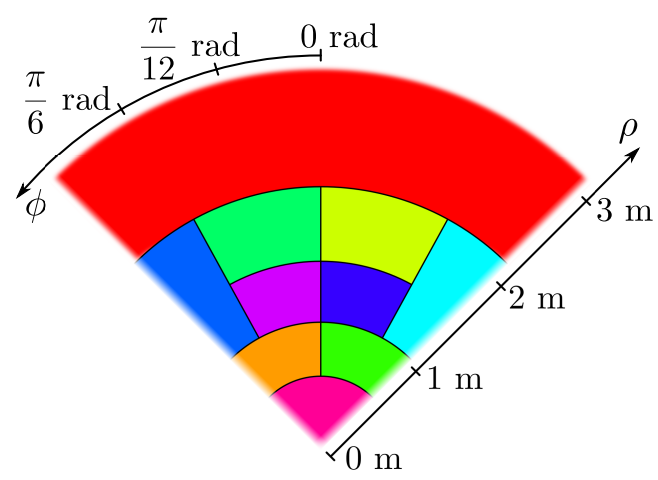

Fig. 5. Resulting Cartesian-space partitions based on the differences of the dual-arm synergies computed from the captured human movements.

\section{MOtion PLANNING}

The dual-arm synergies define a $k$-dimensional box centered at the barycenter of the configurations used to obtain the synergies and with each side aligned with a synergy [20], where $k$ is the number of DOFs of the robot torso (i.e. without considering the translational DOFs of the robot base). In order for the box to contain the $(100-\alpha) \%$ of the configuration distribution for a given $\alpha$ (i.e. any torso configuration inside the box would be then similar to the ones used to compute the synergies), each side of the box is set to $2 \sqrt{2} \operatorname{erf}^{-1}(\sqrt[k]{1-\alpha})$ times the standard deviation of the configurations in the corresponding direction (synergy). The box dimension can be decreased by using only $p<k$ synergies (picking them in order) such that $p$ is the minimum value making the accumulated variance be above a confidence level of $(100-\beta) \%$ for a given $\beta$. Here, $k=13$ due to the hardware and we use $\alpha=\beta=5 \%$. Despite this simplification, the resulting lower-dimensional box $B_{p}$ still represents accurately the mapped torso configurations. Note that since the Cartesian space is partitioned in sectors based on the synergies, each sector may have a different $B_{p}$. Thereby, if the planning of the dual-arm motions is performed in the corresponding $B_{p}$, the planning complexity is reduced and the obtained motions are similar to the movements mapped from the human operator.

The RRT-Connect algorithm [31] has been widely used in motion planning since it obtains good results even in problems with cluttered environments and robots with a lot of DOFs. It maintains two trees of configurations, one rooted at the start configuration $\boldsymbol{q}_{\text {start }}$ and the other rooted at the goal configuration $\boldsymbol{q}_{\text {goal }}$. In each iteration, one of the trees is steered towards a random configuration $\boldsymbol{q}_{\text {rand }}$ by taking a single step of length $\epsilon$ from the closest configuration $\boldsymbol{q}_{\text {near }}$ in the tree, reaching a new configuration $\boldsymbol{q}_{\text {new }}$. If the rectilinear segment connecting $\boldsymbol{q}_{\text {near }}$ and $\boldsymbol{q}_{\text {new }}$ is collision-free, the segment is added to the tree and the other tree takes then successive steps towards $\boldsymbol{q}_{\text {new }}$ until either a collision or $\boldsymbol{q}_{\text {new }}$ is reached. If the latter occurs, the planning process stops since a collision-free path connecting $\boldsymbol{q}_{\text {start }}$ and $\boldsymbol{q}_{\text {goal }}$ already exists trough the trees. Otherwise, the trees swap their roles and this process is repeated until a solution is found or some termination condition is satisfied.

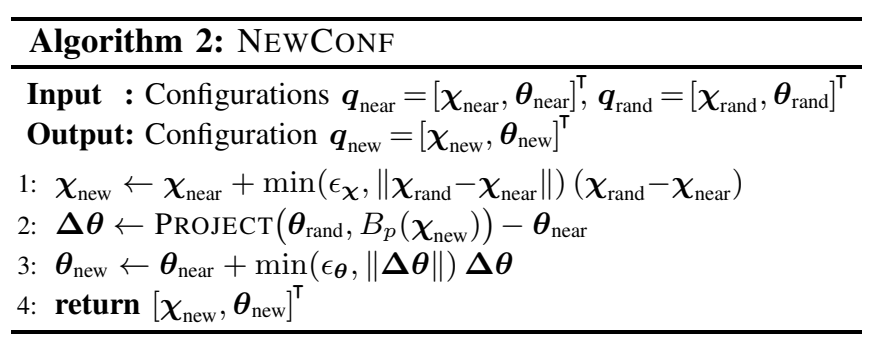

In order to integrate the obtained dual-arm synergies and the Cartesian-space discretization into the motion planning, a modification of the RRT-Connect is proposed. In this work, the standard function used to grow a tree from a given $\boldsymbol{q}_{\text {near }}$ to some $\boldsymbol{q}_{\text {new }}$ is replaced by the function NEWCONF described in Algorithm 2. In this function, the robot position $\chi$ and the torso configuration $\boldsymbol{\theta}$ are treated differently following the next procedure. A step, with a maximum length $\epsilon_{\chi}$, is taken from the robot position $\chi_{\text {near }}$ towards $\chi_{\text {rand }}$, reaching a new robot position $\chi_{\text {new }}$ (Line 1). Then, a step, with a maximum length $\epsilon_{\boldsymbol{\theta}}$, is taken from the torso configuration $\boldsymbol{\theta}_{\text {near }}$ not towards $\boldsymbol{\theta}_{\text {rand }}$ (as it would be done in the standard procedure) but towards its projection onto $B_{p}\left(\chi_{\text {new }}\right)$ (i.e. the lower-dimensional box $B_{p}$ spanned by the synergies of the Cartesian-space region containing $\chi_{\text {new }}$ ), reaching a new torso configuration $\boldsymbol{\theta}_{\text {new }}$ (Lines 2-3). Finally, the computed $\chi_{\text {new }}$ and $\boldsymbol{\theta}_{\text {new }}$ are joined to compose $\boldsymbol{q}_{\text {new }}$ (Line 4).

\section{APPROACH VALIDATION}

For illustrative purposes, the motions of a mobile anthropomorphic dual-arm robot have been planned using the synergies computed from the captured human movements and the planner introduced above. The robot must go from a start configuration in the neighborhood of a table to a goal configuration in which the robot is pre-grasping two cylinders lying on the table (see Fig. 6). The robot must avoid the collisions and perform human-like motions, which do not have to be exactly the movements used to calculate the synergies. The robot motions have been planned with (a) the proposed planner and (b) the standard RRT-Connect, both implemented within the planning and simulation environment The Kautham Project [27] and run in a 2.13-GHz Intel 2, 4-GB RAM PC. A maximum planning time of $30 \mathrm{~s}$ is allowed and if a path is not found within this time, the execution is marked as a failure. After 100 executions, Table I shows the average values of the success rate, the planning time, the number of iterations and collision checks, the rate of valid segments (i.e. the ratio of iterations in which the trees actually grow) and the path length (measured in $\mathcal{C}$ as the weighted sum of accumulated rotated angles of the wheels and the arm joints along the path). A representative solution path obtained with the proposed planner is shown in Fig. 6.

\section{CONCLUSIONS AND FUture WORK}

This paper has proposed a procedure to obtain dual-arm synergies and to discretize the Cartesian space into different annular sectors based on the differences of the synergies associated to each robot position, for a given task. The focus 


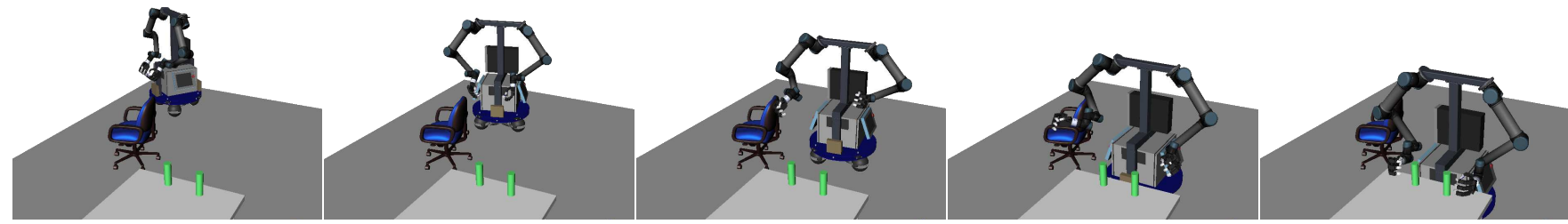

Fig. 6. Snapshots of a planned path for the mobile anthropomorphic dual-arm robot.

TABLE I

AVERAGE RESULTS OF THE MOTION PLANNING USING THE PROPOSED APPROACH (a) AND THE STANDARD RRT-CONNECT (b).

\begin{tabular}{|c|c|c|c|c|c|c|}
\hline \begin{tabular}{|l}
0 \\
$\tilde{Z}$ \\
$\tilde{\Xi}$
\end{tabular} & $\begin{array}{c}\text { Success } \\
\text { rate }\end{array}$ & $\begin{array}{c}\text { Planning } \\
\text { time }\end{array}$ & $\begin{array}{c}\text { \# of } \\
\text { iterations }\end{array}$ & $\begin{array}{c}\# \text { of collision } \\
\text { checks }\end{array}$ & \begin{tabular}{|c|} 
Valid \\
segments
\end{tabular} & $\begin{array}{l}\text { Path } \\
\text { length }\end{array}$ \\
\hline a & $100 \%$ & $2.923 \mathrm{~s}$ & 290 & 2156 & $74.09 \%$ & $4.378 \mathrm{rad}$ \\
\hline $\mathrm{b}$ & $100 \%$ & $11.378 \mathrm{~s}$ & 1940 & 6532 & $63.32 \%$ & $4.731 \mathrm{rad}$ \\
\hline
\end{tabular}

has been set on the approaching movements of a mobile anthropomorphic dual-arm robot previous to a bimanual manipulation task, but the approach is valid for other kind of movements. Thereby, human movements have been captured and mapped to the robot to compute the dual-arm synergies. A likeness function between sets of robot configurations has been used to detect the changes of the synergies and split the Cartesian space accordingly. This Cartesian-space discretization clearly identifies the regions of the free-walk and grasping task phases, as well as their associated synergies. The gradual transition between these two phases is also visible in the discretization. Finally, a motion planner profiting from the synergies and the Cartesian-space partition has been presented as an example.

In the future we plan to extend the proposal to the velocity space while coordinating the robot base, arms and hands all at the same time is an interesting research topic.

\section{REFERENCES}

[1] N. Vahrenkamp, D. Berenson, T. Asfour, J. Kuffner, and R. Dillmann. Humanoid motion planning for dual-arm manipulation and re-grasping tasks. In Proc. IEEE/RSJ Int. Conf. Intelligent Robots and Systems, pages 2464-2470, Oct. 2009.

[2] R. Shauri and K. Nonami. Assembly manipulation of small objects by dual-arm manipulator. Assembly Autom., 31(3):263-274, Aug. 2011.

[3] C. Rodríguez, A. Montaño, and R. Suárez. Planning manipulation movements of a dual-arm system considering obstacle removing. Robotics and Autonomous Systems, 62(12):1816-1826, Aug. 2014.

[4] A.H. Quispe, H. Ben-Amor, and H. Christensen. A taxonomy of benchmark tasks for bimanual manipulators. In Proc. Int. Symp. Robotics Research, pages 1-10, Sept. 2015.

[5] M. Elbanhawi and M. Simic. Sampling-based robot motion planning: A review. IEEE Access, 2:56-77, Jan. 2014.

[6] M. Stilman. Global manipulation planning in robot joint space with task constraints. IEEE Trans. Robot., 26(3):576-584, June 2010.

[7] L. Jaillet, J. Cortés, and T. Siméon. Sampling-based path planning on configuration-space costmaps. IEEE Trans. Robot., 26(4):635-646, Aug. 2010.

[8] N. García, R. Suárez, and J. Rosell. HG-RRT*: Human-Guided optimal random trees for motion planning. In Proc. IEEE Int. Conf. Emerging Technologies and Factory Automation, Sept. 2015.

[9] J. Lee, O. Kwon, L. Zhang, and S.E. Yoon. A selective retractionbased RRT planner for various environments. IEEE Trans. Robotics, 30(4):1002-1011, Aug. 2014.

[10] C. Hu, W. Chen, J. Wang, and H. Wang. Optimal path planning for mobile manipulator based on manipulability and localizability. In Proc. IEEE Int. Conf. Real-time Computing and Robotic, pages 638643, June 2016.
[11] G. Oriolo and C. Mongillo. Motion planning for mobile manipulators along given end-effector paths. In Proc. IEEE Int. Conf. Robotics and Automation, pages 2154-2160, Apr. 2005.

[12] Y. Yang and O. Brock. Elastic roadmaps-motion generation for autonomous mobile manipulation. Auton. Robots, 28(1):113-130, Jan. 2010

[13] M. Liarokapis, C.P. Bechlioulis, P.K. Artemiadis, and K.J. Kyriakopoulos. Deriving humanlike arm hand system poses. J. Mechanisms and Robotics, 9(1), Jan. 2017.

[14] A. Dragan, K. Lee, and S. Srinivasa. Legibility and predictability of robot motion. In ACM/IEEE Int. Conf. Human-Robot Interaction, pages 301-308, Mar. 2013

[15] J. Romero, T. Feix, C.H. Ek, H. Kjellström, and D. Kragic. Extracting postural synergies for robotic grasping. IEEE Trans. Robotics, 29(6):1342-1352, Dec. 2013

[16] S. Sun, C. Rosales, and R. Suárez. Study of coordinated motions of the human hand for robotic applications. In Proc. IEEE Int. Conf. Information and Automation, pages 776-781, June 2010.

[17] J. Rosell, R. Suárez, C. Rosales, and A. Pérez. Autonomous motion planning of a hand-arm robotic system based on captured human-like hand postures. Auton. Robots, 31(1):87-102, July 2011.

[18] D. Prattichizzo, M. Malvezzi, and A. Bicchi M. Gabiccini. On motion and force controllability of precision grasps with hands actuated by soft synergies. IEEE Trans. Robot., 29(6):1440-1456, Aug. 2013.

[19] R. Suárez, J. Rosell, and N. García. Using synergies in dual-arm manipulation tasks. In Proc. IEEE Int. Conf. Robotics and Automation, pages 5655-5661, May 2015.

[20] N. García, R. Suárez, and J. Rosell. Task-Dependent synergies for motion planning of an anthropomorphic dual-arm system. IEEE Trans. Robotics, 33(3):756-764, June 2017.

[21] N. García, J. Rosell, and R. Suárez. Motion planning using firstorder synergies. In Proc. IEEE/RSJ Int. Conf. Intelligent Robots and Systems, pages 2058-2063, Sept. 2015.

[22] N. García, R. Suárez, and J. Rosell. First-Order synergies for motion planning of anthropomorphic dual-arm robots. In Proc. IFAC World Congress, pages 2283-2290, July 2017.

[23] N. García, J. Rosell, and R. Suárez. Motion planning by demonstration with human-likeness evaluation for dual-arm robots. IEEE Trans. Systems, Man, and Cybernetics: Systems, PP(99):1-10, 2017.

[24] W. Chen, C. Xiong, and S. Yue. On configuration trajectory formation in spatiotemporal profile for reproducing human hand reaching movement. IEEE Trans. Cybernetics, 46(3):804-816, Mar. 2016.

[25] M. Sreenivasa, P. Souères, and J.P. Laumond. Walking to grasp: Modeling of human movements as invariants and an application to humanoid robotics. IEEE Trans. Systems, Man and Cybernetics A: Systems and Humans, 42(4):880-893, July 2012.

[26] S. Albrecht, K. Ramirez-Amaro, F. Ruiz-Ugalde, D. Weikersdorfer, M. Leibold, M. Ulbrich, and M. Beetz. Imitating human reaching motions using physically inspired optimization principles. In Proc. IEEE-RAS Int. Conf. Humanoid Robots, pages 602-607, Oct. 2011.

[27] J. Rosell, A. Pérez, A. Aliakbar, Muhayyuddin, L. Palomo, and N. García. The Kautham Project: A teaching and research tool for robot motion planning. In Proc. IEEE Int. Conf. Emerging Technologies and Factory Automation, Sept. 2014.

[28] Du Q. Huynh. Metrics for 3D rotations: Comparison and analysis. $J$. Mathematical Imaging and Vision, 35(2):155-164, Oct. 2009.

[29] W. Chen, C. Xiong, and S. Yue. Mechanical implementation of kinematic synergy for continual grasping generation of anthropomorphic hand. IEEE/ASME Trans. Mechatronics, 20(3):1249-1263, June 2015.

[30] T. Wimböck, B. Jan, and G. Hirzinger. Synergy-level impedance control for a multifingered hand. In Proc. IEEE/RSJ Int. Conf. Intelligent Robots and Systems, pages 973-979, Sept. 2011.

[31] J. J. Kuffner and S. LaValle. RRT-Connect: An efficient approach to single-query path planning. In Proc. IEEE Int. Conf. Robotics and Automation, pages 995-1001, Apr. 2000. 